\title{
Efficacy of slit lamp breath shields
}

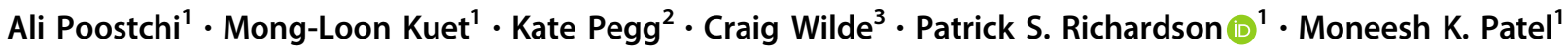

Received: 16 April 2020 / Revised: 24 April 2020 / Accepted: 28 April 2020 / Published online: 12 May 2020

(c) The Royal College of Ophthalmologists 2020

The use of enlarged breath shields has been suggested as part of a wide range of infection control measures implemented during the COVID-19 pandemic. Breath shields have long been a standard feature of slit lamps and act as a physical barrier between the examiner and subject but there is an absence of evidence on their effectiveness in reducing droplet transmission and respiratory infections.

SARS-CoV-2 shares many of the features of other respiratory viruses including SARS-CoV-1 and is thought to be commonly spread though respiratory droplets $(>5 \mu \mathrm{m})$ and fomites [1]. Fomites are formed either from droplets settling on surfaces or through direct contamination from touching mucosal surfaces. Smaller aerosolised droplet nuclei $(\leq 5 \mu \mathrm{m})$ can travel further and remain in air longer. They have been shown to carry viable virus particles in experimental conditions [2] but are not thought to be a common mode of transmission of COVID-19 [1]. The risk of transmission from tears is also thought to be low [3].

We sought to examine the efficacy of facemasks and standard and augmented slit lamp breath shields using a breathing simulator. These have been described previously and generally comprise of a particle source, commonly a nebuliser attached to a bellows or air tank and a particle detector which can consist of a laser particle counter [4] or an impinger from which viral particles can be sampled from air, cultured in cells and detected as plaques [5]. Direct visual inspection of sprayed dye droplets has also been described as a way to test eye protection $[6,7]$. We experimented using nebulised fluorescein $2 \%$ but were

Mong-Loon Kuet

mkuet@nhs.net

1 Department of Ophthalmology, Royal Derby Hospital, University Hospitals of Derby and Burton NHS Foundation Trust, Derby, UK

2 Pharmacy Quality Assurance, Royal Derby Hospital, University Hospitals of Derby and Burton NHS Foundation Trust, Derby, UK

3 Department of Ophthalmology, Queen's Medical Centre, Nottingham University Hospitals NHS Trust, Nottingham, UK unable to capture sufficient dye to determine the patterns of droplet distribution.

We used a mouthpiece nebuliser (Galemed Corp, Taiwan) containing $5 \mathrm{ml}$ of $0.9 \%$ saline as our particle source and attached it to a $500 \mathrm{ml}$ paediatric bag valve mask that was manually compressed 12 times per minute to simulate normal adult tidal breathing. The device produces a range of particles from 1 to $25 \mu \mathrm{m}$ with median mass aerodynamic diameter of $3.8 \mu \mathrm{m}$. We used a Met One A2400 optical particle counter (Hach Co, Loveland, CO) operating at a flow rate of one cubic foot per minute to detect particles that reached the eyepiece over a 1-min period. This was initially performed without any shielding, and then repeated with the standard $(11 \times 11 \times$ $0.2 \mathrm{~cm})$ and augmented $(45 \times 44 \times 0.2 \mathrm{~cm})$ acrylic shields attached to the slit lamp objective lens (Fig. 1). We then tested the effect of placing a fluid resistant surgical facemask (BARRIER 4313, Mölnlycke Healthcare, Sweden) over the nebuliser mouthpiece alone and in combination with the large shield. The slit lamp arm was offset to $60^{\circ}$ throughout and each barrier was tested five times. Linear regression was used to determine the effect of shield type and particle size on particle count. All analyses were performed using Stata v14.

With no shield in place, the mean log particle count was 3.59 (95\% CI: 3.48-3.70). There was a significant reduction to 3.01 (95\% CI: $2.90-3.13, p<0.01)$ with the standard

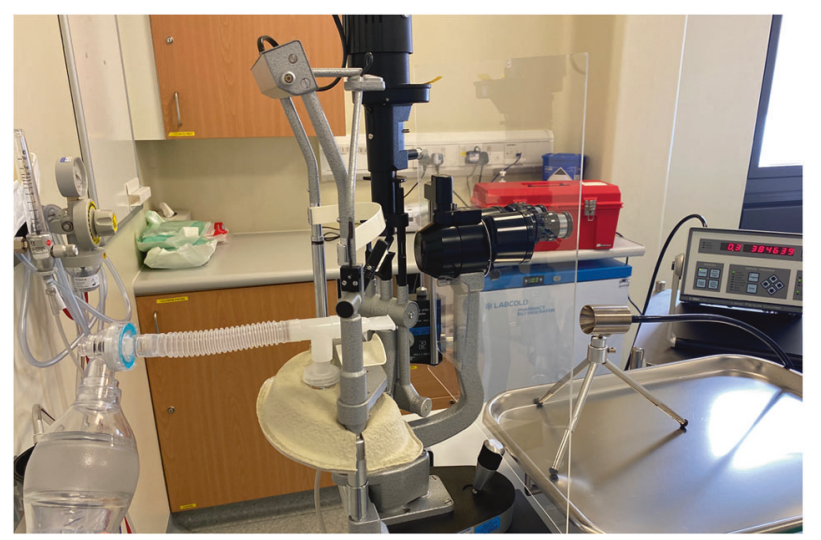

Fig. 1 Photograph of the experimental setup. The nebuliser particle source and optical particle detector can be seen either side of the slit lamp. 


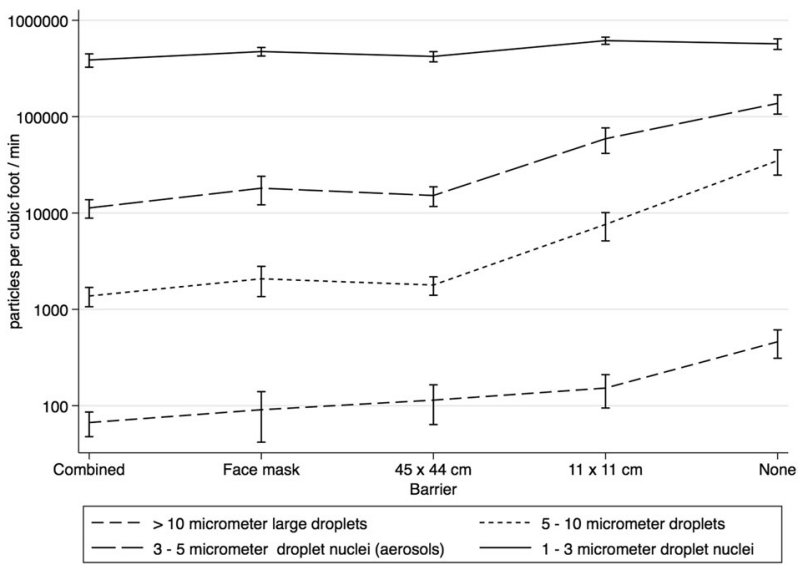

Fig. 2 Mean particle counts $(N=5)$ over $1 \mathrm{~min}$, including $95 \%$ confidence intervals. Particles counts are stratified by size and shown against the different types of barrier used. The combined effect of the surgical facemask and large shield when used together is also shown.

shield, 2.63 (95\% CI: $2.52-2.75, p<0.01$ ) with the augmented shield and 2.62 (95\% CI: $2.50-2.73, p<0.01)$ with the facemask secured in front of the nebuliser. With the mask and large shield both in place, the mean log count dropped to 2.47 (95\% CI: $2.36-2.59, p<0.01$ ).

We found that the barriers we tested were all effective at reducing transmission of particles $>3 \mu \mathrm{m}$. The surgical facemask and large shield offered the best protection, while the small standard shield seemed to be effective for larger particles (Fig. 2). The benefits of larger shields should be weighed against the increased risk of fomite transmission, especially where they cause the user to touch the sides in order to perform tonometry or manipulate the slit lamp arm. Barrier protection, while helpful, should be seen as only one part of a combined approach with hand hygiene and effective cleaning essential to limit viral spread. There is ongoing debate over the use of masks. Evidence from a large-clusterbased randomised control trial showed no benefit from using N95 masks compared to surgical facemasks to reduce influenza and other respiratory viral illnesses [8]. Subsequent meta-analyses have shown similar results with the benefits of filtering facepiece (FFP) masks thought to be offset by discomfort with their prolonged use leading to more frequent manipulation and/or reduced compliance [9]. There may be a similar tradeoff between different sizes of shield with an increased risk of contamination through contact with larger shields.

The protocol we describe has helped us to reduce some of the uncertainty that surrounds our use of protective equipment and can be adapted to different scenarios. Most of the components we used were readily available and the particle counter was not difficult to source. Particle counters are routinely used in our trust to monitor sterility of theatres and pharmacy clean rooms and more recently to test the fit of FFP masks. A limitation of our approach is that we did not distinguish between infectious and noninfectious particles or their source. We performed our study in a room used for intravitreal injections to limit the number of background particles but this remains a potential confounder. We found it reassuring that the standard measures in place were effective and that the larger shield and facemask appeared to offer some additional protection. Despite the close proximity of slit lamp examinations, the effectiveness of shielding together with the short contact time and lack of aerosol generation, leads us to expect the risk of transmission from this route to be low. Viruses spread fear and uncertainty along with disease. Through research, consultation and pragmatic use of evidence, we hope to help build a shield to this as well.

Acknowledgements We are grateful to Stuart Lawrie for the construction and installation of the augmented acrylic breath shields in our department.

\section{Compliance with ethical standards}

Conflict of interest The authors declare that they have no conflict of interest.

Publisher's note Springer Nature remains neutral with regard to jurisdictional claims in published maps and institutional affiliations.

\section{References}

1. World Health Organization. Report of the WHO-China joint mission on coronavirus disease 2019 (COVID-19). Geneva: World Health Organization; 2020.

2. van Doremalen N, Bushmaker T, Morris DH, Holbrook MG, Gamble A, Williamson BN, et al. Aerosol and surface stability of SARS-CoV-2 as compared with SARS-CoV-1. N Engl J Med. 2020;382:1564-7.

3. Seah IYJ, Anderson DE, Kang AEZ, Wang L, Rao P, Young BE, et al. Assessing viral shedding and infectivity of tears in coronavirus disease 2019 (COVID-19) patients. Ophthalmology. 2020.

4. Lindsley WG, King WP, Thewlis RE, Reynolds JS, Panday K, Cao $\mathrm{G}$, et al. Dispersion and exposure to a cough-generated aerosol in a simulated medical examination room. J Occup Environ Hyg. 2012; 9:681-90.

5. Makison Booth C, Clayton M, Crook B, Gawn JM. Effectiveness of surgical masks against influenza bioaerosols. J Hosp Infect. 2013;84:22-6.

6. Mansour AA, Even JL, Phillips S, Halpern JL. Eye protection in orthopaedic surgery. An in vitro study of various forms of eye protection and their effectiveness. J Bone Joint Surg Am. 2009;91:1050-4.

7. Canelli R, Connor CW, Gonzalez M, Nozari A, Ortega R. Barrier enclosure during endotracheal intubation. N Engl J Med. 2020.

8. Radonovich LJ, Simberkoff MS, Bessesen MT, Brown AC, Cummings DAT, Gaydos CA, et al. N95 respirators vs medical masks for preventing influenza among health care personnel: a randomized clinical trial. JAMA. 2019;322:824-33.

9. Long Y, Hu T, Liu L, Chen R, Guo Q, Yang L, et al. Effectiveness of N95 respirators versus surgical masks against influenza: a systematic review and meta-analysis. J Evid Based Med. 2020. 